\title{
WEAK ORDER FOR THE DISCRETIZATION OF THE STOCHASTIC HEAT EQUATION
}

\author{
ARNAUD DEBUSSCHE AND JACQUES PRINTEMS
}

\begin{abstract}
In this paper we study the approximation of the distribution of $X_{t}$ Hilbert-valued stochastic process solution of a linear parabolic stochastic partial differential equation written in an abstract form as

$$
\mathrm{d} X_{t}+A X_{t} \mathrm{~d} t=Q^{1 / 2} \mathrm{~d} W(t), \quad X_{0}=x \in H, \quad t \in[0, T],
$$

driven by a Gaussian space time noise whose covariance operator $Q$ is given. We assume that $A^{-\alpha}$ is a finite trace operator for some $\alpha>0$ and that $Q$ is bounded from $H$ into $D\left(A^{\beta}\right)$ for some $\beta \geq 0$. It is not required to be nuclear or to commute with $A$.

The discretization is achieved thanks to finite element methods in space (parameter $h>0$ ) and a $\theta$-method in time (parameter $\Delta t=T / N)$. We define a discrete solution $X_{h}^{n}$ and for suitable functions $\varphi$ defined on $H$, we show that

$$
\left|\mathbb{E} \varphi\left(X_{h}^{N}\right)-\mathbb{E} \varphi\left(X_{T}\right)\right|=O\left(h^{2 \gamma}+\Delta t^{\gamma}\right)
$$

where $\gamma<1-\alpha+\beta$. Let us note that as in the finite dimensional case the rate of convergence is twice the one for pathwise approximations.
\end{abstract}

\section{INTRODUCTION}

In this article, we study the convergence of the distributions of numerical approximations of the solutions of a large class of linear parabolic stochastic partial differential equations. The numerical analysis of stochastic partial differential equations has been recently the subject of many articles. (See among others 11, 7], 11, 12, 13, 14, 15, 16, 17, 18, 19, 24, 25, 28, 29, 35, 33, 34]). In all of these papers, the aim is to give an estimate on the strong order of convergence for a numerical scheme. In other words, on the order of pathwise convergence. It is well known that in the case of stochastic differential equations in finite dimension, the so-called weak order is much better than the strong order. The weak order is the order of convergence of the law of approximations to the true solution. For instance, the standard Euler scheme is of strong order 1/2 for the approximation of a stochastic differential equation while the weak order is 1. A basic tool to study the weak order is the Kolmogorov equation associated to the stochastic equation (see [22, 26], 27] 31]).

In infinite dimension, this problem has been studied in fewer articles. In 3 , the case of a stochastic delay equation is studied. To our knowledge, only [8, 20] and more recently 9] consider stochastic partial differential equations. In 8 the nonlinear Schrödinger equations are considered. In the present article, we consider

Received by the editor October 30, 2007 and, in revised form, May 7, 2008.

2000 Mathematics Subject Classification. Primary 60H15, 60H35, 65C30, 65M60.

Key words and phrases. Weak order, stochastic heat equation, finite element, Euler scheme.

(C)2008 American Mathematical Society Reverts to public domain 28 years from publication 
the case of the full discretization of a parabolic equation. We restrict our attention to a linear equation with additive noise which contains several difficulties. The general case of semilinear equations with state dependent noise presents further difficulties and will be treated in a forthcoming article. This case is treated in [20] but there only finite dimensional functionals of the solution are used and the finite dimensional method can be used. The article 9] presents a similar result to ours but a restriction on the functional is also imposed. However, it is much weaker than in 20].

Note that there are essential differences between the equations treated in [3] and 8]. Indeed, no spatial difference operator appears in a delay equation. In the case of the Schrödinger equation the linear evolution defines a group and it is possible to get rid of the differential operator by inverting the group. Furthermore, in [8], the data are assumed to be very smooth.

In this article, we get rid of the differential operator by using a similar trick as in [8]. However, since the linear evolution operator is not invertible, this introduces extra difficulties. Moreover, we consider a full discretization using an implicit Euler scheme and finite elements for the spatial discretization. We give an estimate of the weak order of convergence with minimal regularity assumptions on the data. In fact, we show that as in the finite dimensional case the weak order is twice the strong order of convergence, both in time and space.

In dimension $d=1,2,3$, let us consider the following stochastic partial differential equation:

$$
\frac{\partial u(x, t)}{\partial t}-\Delta u(x, t)=\dot{\eta}(x, t)
$$

where $x \in \mathcal{O}$, a bounded open set of $\mathbb{R}^{d}$, and $\left.\left.t \in\right] 0, T\right]$, with Dirichlet boundary conditions and initial data and $\dot{\eta}=\frac{\partial \eta}{\partial t}$ with $\eta$ denotes a real-valued Gaussian process. It is convenient to use an abstract framework to describe the noise more precisely. Let $W$ be a cylindrical Wiener process on $L^{2}(\mathcal{O})$; in other words, $\frac{\partial W}{\partial t}$ is the space time white noise. Equivalently, given an orthonormal basis $\left(e_{i \in \mathbb{N}}\right)$ of $L^{2}(\mathcal{O}), W$ has the expansion

$$
W(t)=\sum_{i \in \mathbb{N}} \beta_{i}(t) e_{i}
$$

where $\left(\beta_{i}\right)_{i \in \mathbb{N}}$ is a family of independent standard Brownian motions (see section 2.4 below). We consider noises of the form $\eta(t)=Q^{1 / 2} W(t)$ where $Q$ is a nonnegative symmetric bounded linear operator on $L^{2}(\mathcal{O})$. For example 1 , given a function $q$ defined on $\mathcal{O}$, we can take

$$
\eta(x, t)=\int_{\mathcal{O}} q(x-y) W(y, t) \mathrm{d} y,
$$

Then the process $\eta$ has the following correlation function:

$$
\mathbb{E} \eta(x, t) \eta(y, s)=c(x-y)(t \wedge s) \quad \text { with } c(r)=\int_{\mathcal{O}} q(z+r) q(z) \mathrm{d} z .
$$

The operator $Q$ is then given by $Q f(x)=\int_{\mathcal{O}} c(x-y) f(y) \mathrm{d} y$. Note that if the $q$ is the Dirac mass at $0, \eta=W$ and $Q=I$.

\footnotetext{
${ }^{1}$ The equations describing this example are formal, it is not difficult to give a rigourous meaning. This is not important in our context. Note that $q$ does not need to be a function and a distribution is allowed.
} 
Let us also set $A=-\Delta, D(A)=H^{2}(\mathcal{O}) \cap H_{0}^{1}(\mathcal{O})$ and $H=L^{2}(\mathcal{O})$. Then $A: D(A) \rightarrow H$ can be seen as an unbounded operator on $H$ with domain $D(A)$. Our main assumption concerning $Q$ is that $A^{\sigma / 2} Q$ defines a bounded operator on $L^{2}(\mathcal{O})$ with $\sigma>-1 / 2$ if $d=1, \sigma>0$ if $d=2$ and $\sigma>1 / 2$ if $d=3$. In the example above, this amounts to requiring that $(-\Delta)^{\sigma / 2} q \in L^{2}(\mathcal{O})$. It is well known that these conditions are sufficient to ensure the existence of continuous solutions of (1.1).

If we write $u(t)=u(\cdot, t)$ seen as a $H$-valued stochastic process, then (1.1) can be rewritten under the abstract Ito form

$$
\mathrm{d} u(t)+A u(t) \mathrm{d} t=Q^{1 / 2} \mathrm{~d} W(t) .
$$

In this article, we consider such an abstract equation and study the approximation of the law of the solutions of (1.2) by means of the $\theta$-method and finite elements. Let $\left\{V_{h}\right\}_{h \geq 0}$ be a family of finite dimensional subspaces of $D\left(A^{1 / 2}\right)$. Let $N \geq 1$ be an integer and $\Delta t=T / N$. The numerical scheme is given by

$$
\left(u_{h}^{n+1}-u_{h}^{n}, v_{h}\right)+\Delta t\left(A u_{h}^{n+\theta}, v_{h}\right)=\sqrt{\Delta t}\left(Q^{1 / 2} \chi^{n+1}, v_{h}\right),
$$

for any $v_{h} \in V_{h}$, where $\sqrt{\Delta t} \chi^{n+1}=W((n+1) \Delta t)-W(n \Delta t)$ is the noise increment and where $(\cdot, \cdot)$ is the inner product of $H$. The unknown is approximated at time $n \Delta t, 0 \leq n \leq N$ by $u_{h}^{n} \in V_{h}$. In (1.3), we have used the notation $u_{h}^{n+\theta}$ for $\theta u_{h}^{n+1}+(1-\theta) u_{h}^{n}$ for some $\theta \in(1 / 2,1]$. We prove that an error estimate of the form

$$
\left|\mathbb{E}(\varphi(u(n \Delta t)))-\mathbb{E}\left(\varphi\left(u_{h}^{n}\right)\right)\right| \leq c\left(h^{2 \gamma}+\Delta t^{\gamma}\right)
$$

for any function $\varphi$ which is $C^{2}$ and bounded on $H$. With the above notation, $\gamma$ is required to be strictly less than $1-d / 2+\sigma / 2$. This is exactly twice the strong order (see [33, 35]). If $d=1$ and $\sigma=0$, the condition is $\gamma<1 / 2$ and we obtain a weak order which is $1 / 2$ in time and 1 in space.

\section{Preliminaries}

2.1. Functional spaces. It is convenient to change the notation and rewrite the unknown of (1.2) as $X$. We thus consider the following stochastic partial differential equation written in the abstract form

$$
\mathrm{d} X_{t}+A X_{t} \mathrm{~d} t=Q^{1 / 2} \mathrm{~d} W(t), \quad X_{0}=x \in H, \quad 0<t \leq T,
$$

where $H$ is a Hilbert space whose inner product is denoted by $(\cdot, \cdot)$ and its associated norm by $|\cdot|$, the process $\left\{X_{t}\right\}_{t \in[0, T]}$ is an $H$-valued stochastic process, $A$ a nonnegative self-adjoint unbounded operator on $H$ whose domain $D(A)$ is dense in $H$ and compactly embedded in $H, Q$ a nonnegative symmetric operator on $H$ and $\{W(t)\}_{t \in[0, T]}$ a cylindrical Wiener process on $H$ adapted to a given normal filtration $\left\{\mathcal{F}_{t}\right\}_{t \in[0, T]}$ in a given probability space $(\Omega, \mathcal{F}, \mathbb{P})$.

It is well known that there exists a sequence of nondecreasing positive real numbers $\left\{\lambda_{n}\right\}_{n \geq 1}$ together with $\left\{e_{n}\right\}_{n \geq 1}$ a Hilbertian basis of $H$ such that

$$
A e_{n}=\lambda_{n} e_{n} \quad \text { with } \lim _{n \rightarrow \infty} \lambda_{n}=+\infty .
$$

We set for any $s \geq 0$,

$$
D\left(A^{s / 2}\right)=\left\{u=\sum_{n=1}^{\infty}\left(u, e_{n}\right) e_{n} \in H \quad \text { such that } \sum_{n=1}^{\infty} \lambda_{n}^{s}\left(u, e_{n}\right)^{2}<\infty\right\},
$$


and

$$
A^{s} u=\sum_{n=1}^{\infty} \lambda_{n}^{s}\left(u, e_{n}\right) e_{n}, \quad \forall u \in D\left(A^{s}\right)
$$

It is clear that $D\left(A^{s / 2}\right)$ endowed with the norm $u \mapsto\|u\|_{s}:=\left|A^{s / 2} u\right|$ is a Hilbert space. We define also $D\left(A^{-s / 2}\right)$ with $s \geq 0$ as the completed space of $H$ for the topology induced by the norm $\|u\|_{-s}=\sum_{n=1}^{\infty} \lambda_{n}^{-s}\left(u, e_{n}\right)^{2}$. In this case $D\left(A^{-s / 2}\right)$ can be identified with the topological dual of $D\left(A^{s / 2}\right)$, i.e. the space of the linear forms on $D\left(A^{s / 2}\right)$ which are continuous with respect to the topology induced by the norm $\|\cdot\|_{s}$.

Moreover, these spaces can be obtained by interpolation between them. Indeed, for any reals $s_{1} \leq s \leq s_{2}$, one has the continuous embeddings $D\left(A^{s_{2} / 2}\right) \subset$ $D\left(A^{s / 2}\right) \subset D\left(A^{s_{1} / 2}\right)$ and by Hölder inequality

$$
\|u\|_{s} \leq\|u\|_{s_{1}}^{1-\lambda}\|u\|_{s_{2}}^{\lambda}, \quad s=(1-\lambda) s_{1}+\lambda s_{2}
$$

for any $u \in D\left(A^{s_{2} / 2}\right)$.

We denote by $\|\cdot\|_{X}$ the norm of a Banach space $X$. If $X$ and $Y$ denote two Banach spaces, we denote by $\mathcal{L}(X, Y)$ the Banach space of bounded linear operators from $X$ into $Y$ endowed with the norm $\|B\|_{\mathcal{L}(X, Y)}=\sup _{x \in X}\|B x\|_{Y} /\|x\|_{X}$. When $X=Y$, we use the shorter notation $\mathcal{L}(X)$.

If $L \in \mathcal{L}(H)$ is a nuclear operator, $\operatorname{Tr}(L)$ denotes the trace of the operator $L$, i.e.

$$
\operatorname{Tr}(L)=\sum_{i=1}^{\infty}\left(L e_{i}, e_{i}\right)<+\infty .
$$

It is well known that the previous definition does not depend on the choice of the Hilbertian basis. Moreover, the following properties hold for a nuclear operator $L$

$$
\operatorname{Tr}(L M)=\operatorname{Tr}(M L), \quad \text { for any } M \in \mathcal{L}(H),
$$

and if $L$ is nonnegative

$$
\operatorname{Tr}(L M) \leq \operatorname{Tr}(L)\|M\|_{\mathcal{L}(H)}, \quad \text { for any } M \in \mathcal{L}(H) .
$$

Hilbert-Schmidt operators also play an important role. Given two Hilbert spaces $K_{1}, K_{2}$, an operator $L \in \mathcal{L}\left(K_{1}, K_{2}\right)$ is Hilbert-Schmidt if $L^{*} L$ is a nuclear operator on $K_{1}$ or equivalently if $L L^{*}$ is nuclear on $K_{2}$. We denote by $\mathcal{L}_{2}\left(K_{1}, K_{2}\right)$ the space of such operators. It is a Hilbert space for the norm

$$
\|L\|_{\mathcal{L}_{2}\left(K_{1}, K_{2}\right)}=\left(\operatorname{Tr}\left(L^{*} L\right)\right)^{1 / 2}=\left(\operatorname{Tr}\left(L L^{*}\right)\right)^{1 / 2} .
$$

It is classical that, given four Hilbert spaces $K_{1}, K_{2}, K_{3}, K_{4}$, if $L \in \mathcal{L}_{2}\left(K_{2}, K_{3}\right)$, $M \in \mathcal{L}\left(K_{1}, K_{2}\right), N \in \mathcal{L}\left(K_{3}, K_{4}\right)$, then $N L M \in \mathcal{L}_{2}\left(K_{1}, K_{4}\right)$ and

$$
\|N L M\|_{\mathcal{L}_{2}\left(K_{1}, K_{4}\right)} \leq\|N\|_{\mathcal{L}\left(K_{3}, K_{4}\right)}\|L\|_{\mathcal{L}_{2}\left(K_{2}, K_{3}\right)}\|M\|_{\mathcal{L}\left(K_{1}, K_{2}\right)} .
$$

See [5], appendix C, or [10] for more details on nuclear and Hilbert-Schmidt operators.

If $X$ is a Banach space, we denote by $\mathcal{C}_{b}(H ; X)$ the Banach space of $X$-valued, continuous and bounded functions on $H$. We also denote by $C_{b}^{k}(H)$ the space of $k$-times continuously differentiable real-valued functions on $H$. The first order differential of a function $\varphi \in C_{b}^{1}(H)$ is identified with its gradient and is then considered as an element of $C_{b}(H ; H)$. It is denoted by $D \varphi$. Similarly, the second order differential of a function $\varphi \in C_{b}^{2}(H)$ is seen as a function from $H$ into the Banach space $\mathcal{L}(H)$ and is denoted by $D^{2} \varphi$. 
2.2. The deterministic stationary problem. We need some classical results on the deterministic stationary version of (2.4). In this case, special attention has to be paid to the space $V=D\left(A^{1 / 2}\right) \subset H$. It is a Hilbert space whose embedding into $H$ is dense and continuous. Its inner product is denoted by $((\cdot, \cdot))$. We have

$$
\begin{aligned}
((u, v)) & =\left(A^{1 / 2} u, A^{1 / 2} v\right), & & \text { for any } u \in V, v \in V \\
& =(A u, v), & & \text { for any } u \in D(A), v \in H .
\end{aligned}
$$

Then by a density argument and the uniqueness of the Riesz representation (in $V$ ) we conclude that $A$ is invertible from $V$ into $V^{\prime}=D\left(A^{-1 / 2}\right)$ or from $D(A)$ into $H$. We will set $T=A^{-1}$ its inverse. It is bounded and positive on $H$ and on $V$.

For any $f \in H, u=T f$ is by definition the unique solution of the following problem:

$$
u \in V, \quad((u, v))=(f, v), \quad \text { for any } v \in V .
$$

Let $\left\{V_{h}\right\}_{h>0}$ be a family of finite dimensional subspaces of $V$ parametrized by a small parameter $h>0$. For any $h>0$, we denote by $P_{h}\left(\right.$ resp. $\left.\Pi_{h}\right)$ the orthogonal projector from $H$ (resp. $V$ ) onto $V_{h}$ with respect to the inner product $(\cdot, \cdot)$ (resp. $((\cdot, \cdot)))$.

For any $h>0$, we denote by $A_{h}$ the linear bounded operator from $V_{h}$ into $V_{h}$ defined by

$$
\left(\left(u_{h}, v_{h}\right)\right)=\left(A_{h} u_{h}, v_{h}\right)=\left(A u_{h}, v_{h}\right) \quad \text { for any } u_{h} \in V_{h}, v_{h} \in V_{h} .
$$

It is clear that $A_{h}: V_{h} \rightarrow V_{h}$ is also invertible. Its inverse is denoted by $T_{h}$. For any $f \in H, u_{h}=T_{h} f$ is by definition the solution of the following problem:

$$
u_{h} \in V_{h}, \quad\left(\left(u_{h}, v_{h}\right)\right)=\left(f, v_{h}\right)=\left(P_{h} f, v_{h}\right), \quad \text { for any } v_{h} \in V_{h} .
$$

It is also clear that $A_{h}$ and $T_{h}$ are positive definite symmetric bounded linear operators on $V_{h}$. We denote by $\left\{\lambda_{i, h}\right\}_{1 \leq i \leq I(h)}$ the sequence of its nonincreasing positive eigenvalues and $\left\{e_{i, h}\right\}_{1 \leq i \leq I(h)}$ the associated orthonormal basis of $V_{h}$ of its eigenvectors. Again, by Hölder inequality, $A_{h}$ satisfies the following interpolation inequality:

$$
\left|A_{h}^{s} u_{h}\right| \leq\left|A_{h}^{s_{1}} u_{h}\right|^{\lambda}\left|A_{h}^{s_{2}} u_{h}\right|^{1-\lambda}, u_{h} \in V_{h}, s=\lambda s_{1}+(1-\lambda) s_{2} .
$$

The consequences of (2.11) are summarized in the following lemma.

Lemma 2.1. Let $A_{h} \in \mathcal{L}\left(V_{h}\right)$, defined in (2.11). Let $T$ and $T_{h}$ be as defined in (2.10) and (2.12). Then the following hold for any $w_{h} \in V_{h}$ and $v \in V$ :

$$
\begin{aligned}
T_{h} P_{h} & =\Pi_{h} T, \\
\left|A^{1 / 2} w_{h}\right| & =\left|A_{h}^{1 / 2} w_{h}\right|, \\
\left|T_{h}^{1 / 2} w_{h}\right| & =\left|T^{1 / 2} w_{h}\right|, \\
\left|T_{h}^{1 / 2} P_{h} v\right| & \leq\left|T^{1 / 2} v\right| .
\end{aligned}
$$

Proof. Now let $f \in H$. We consider the two solutions $u$ and $u_{h}$ of (2.10) and (2.12). Since $V_{h} \subset V$, we can write (2.10) with $v_{h} \in V_{h}$. Then, substracting we get $\left(\left(u-u_{h}, v_{h}\right)\right)=0$. Hence, $u_{h}=\Pi_{h} u$ the $V$-orthogonal projection of $u$ onto $V_{h}$, i.e. $T_{h} P_{h} f=\Pi_{h} T f$. 
Equation (2.15) follows immediately from the definition (2.11) of $A_{h}$. We now prove (2.17). Equations (2.14) and (2.15) imply

$$
\left|A_{h}^{-1 / 2} P_{h} v\right|=\left\|T_{h} P_{h} v\right\|=\left\|\Pi_{h} T v\right\| \leq\|T v\|=\left|A^{-1 / 2} v\right|,
$$

since $\Pi_{h}: V \rightarrow V_{h}$ is an orthogonal projection for the inner product $\|\cdot\|$.

As regards (2.16), on one hand (2.17) with $v=w_{h} \in V_{h} \subset V$ gives the first inequality $\left|T_{h}^{1 / 2} w_{h}\right| \leq\left|T^{1 / 2} w_{h}\right|$. On the other hand, by (2.11),

$$
\left|\left(A_{h} u_{h}, v_{h}\right)\right|=\left|\left(A u_{h}, v_{h}\right)\right| \leq\left|A^{1 / 2} u_{h}\right|\left|A^{1 / 2} v_{h}\right| .
$$

So $A_{h} u_{h}$ can be considered as a continuous linear form on $D\left(A^{1 / 2}\right)$, i.e. it belongs to $D\left(A^{-1 / 2}\right)$, and

$$
\left|A^{-1 / 2} A_{h} u_{h}\right| \leq\left|A^{1 / 2} u_{h}\right|=\left|A_{h}^{1 / 2} u_{h}\right| .
$$

Taking $u_{h}=A_{h}^{-1} w_{h}$ gives $\left|A^{-1 / 2} w_{h}\right| \leq\left|A_{h}^{-1 / 2} w_{h}\right|$. Equation (2.16) follows.

Our main assumption concerning the spaces $V_{h}$ is that the corresponding linear elliptic problem (2.12) admits an $O\left(h^{r}\right)$ error estimate in $H$ and $O\left(h^{r-1}\right)$ in $V$ for some $r \geq 2$. It is classical to verify that these estimates hold if we suppose that $\Pi_{h}$ satisfies for some constant $\kappa_{0}>0$,

$$
\begin{aligned}
\left|\Pi_{h} v-v\right| & \leq \kappa_{0} h^{s}\left|A^{s / 2} v\right|, \quad 1 \leq s \leq r, \\
\left|A^{1 / 2}\left(\Pi_{h} w-w\right)\right| & \leq \kappa_{0} h^{s^{\prime}-1}\left|A^{s^{\prime} / 2} w\right|, \quad 1 \leq s^{\prime} \leq r-1,
\end{aligned}
$$

where $v \in D\left(A^{s / 2}\right)$ and $w \in D\left(A^{s^{\prime} / 2}\right)$.

Finite elements satisfying these conditions are, for example, $P_{k}$ triangular elements on a convex polygonal domain or $Q_{k}$ rectangular finite element on a rectangular domain provided $k \geq 1$. Approximation by splines can also be considered. (See [4], 30]).

2.3. The deterministic evolution problem. We recall now some results about the spatial discretization of the solution of the deterministic linear parabolic evolution equation:

$$
\frac{\partial u(t)}{\partial t}+A u(t)=0, \quad u(0)=y,
$$

by the finite dimensional one:

$$
\frac{\partial u_{h}(t)}{\partial t}+A_{h} u_{h}(t)=0, \quad u_{h}(0)=P_{h} y \in V_{h} .
$$

It is well known that, under our assumptions, (2.20) defines a contraction semigroup on $H$ denoted by $S(t)=e^{-t A}$ for any $t \geq 0$. Its solution can be read as $u(t)=S(t) y$ where $t \geq 0$. The main properties of $S(t)$ (contraction, regularization) are summed up below:

$$
\left|e^{-t A} x\right| \leq|x|, \quad \text { for any } x \in H,
$$

and

$$
\left|A^{s} e^{-t A} x\right| \leq C(s) t^{-s}|x|,
$$

for any $t>0, s \geq 0$ and $x \in H$. Such a property is based on the definition of $A^{s}$ and the following well known inequality

$$
\sup _{x \geq 0} x^{\varepsilon} e^{-t x} \leq C(\varepsilon) t^{-\varepsilon}, \quad \text { for any } t>0 .
$$


In the same manner, we denote by $S_{h}(t)=e^{-t A_{h}}$ the semi-group on $V_{h}$ such that $u_{h}(t)=S_{h}(t) P_{h} y$, for any $t \geq 0$.

We have various types of convergence of $u_{h}$ towards $u$ depending on the regularity of the initial data $y$. The optimal rates of convergences remain the same as in the corresponding stationary problems (see (2.18)-(2.19)). The estimates are not uniform in time near $t=0$ since the regularization of $S(t)$ is used to prove them. The following lemma gives two classical properties needed in this article.

Lemma 2.2. Let $r \geq 2$ be such that (2.18) and (2.19) hold and $q, q^{\prime}, s, s^{\prime}$ such that $0 \leq s \leq q \leq r, s^{\prime} \geq 0,1 \leq q^{\prime}+s^{\prime} \leq r-1$ and $q^{\prime}<2$. Then there exists constants $\kappa_{i}>0, i=1,2$ independent on $h$ such that for any time $t>0$, one has:

$$
\begin{aligned}
\left\|S_{h}(t) P_{h}-S(t)\right\|_{\mathcal{L}\left(D\left(A^{s / 2}\right), H\right)} & \leq \kappa_{1} h^{q} t^{-(q-s) / 2}, \\
\left\|S_{h}(t) P_{h}-S(t)\right\|_{\mathcal{L}\left(D\left(A^{s^{\prime} / 2}\right), D\left(A^{1 / 2}\right)\right)} & \leq \kappa_{2} h^{q^{\prime}+s^{\prime}} t^{-\left(q^{\prime}+1\right) / 2} .
\end{aligned}
$$

The proof of (2.24) can be found in [2] (see also 32, Theorem 3.5, p. 45). The proof of (2.25) can be found in [21, Theorem 4.1, p. 342 (with $f=0$ ). In fact, we use only $s=s^{\prime}=0$ and $q^{\prime}=1$ below.

2.4. Infinite dimensional stochastic integrals. In this section, we recall basic results on the stochastic integral with respect to the cylindrical Wiener process $W$. More details can be found for instance in [5].

It is well known that $W$ has the expansion

$$
W=\sum_{i=1}^{\infty} \beta_{i} e_{i}
$$

where $\left\{\beta_{i}\right\}_{i \geq 1}$ denotes a family of real-valued mutually independent Brownian motions on $\left(\Omega, \mathcal{F}, \mathbb{P},\left\{\mathcal{F}_{t}\right\}_{t \geq 0}\right)$. The sum does not converge in $H$ and this reflects the bad regularity property of the cylindrical Wiener process. However, it converges a.s. and in $L^{p}(\Omega ; U), p \geq 1$, for any space $U$ such that $H \subset U$ with a HilbertSchmidt embedding. If $\bar{H}=L^{2}(\mathcal{O}), \mathcal{O} \subset \mathbb{R}^{d}$ is open and bounded, we can take $U=H^{-s}(\mathcal{O}), s>d / 2$.

Such a Wiener process can be characterized by

$$
\mathbb{E}(W(t), u)(W(s), v)=t \wedge s(u, v)
$$

for any $t, s \geq 0$ and $u, v \in H$.

Given any predictable operator-valued function $t \mapsto \Phi(t), t \in[0, T]$, it is possible to define $\int_{0}^{T} \Phi(s) \mathrm{d} W(s)$ in a Hilbert space $K$ if $\Phi$ takes values in $\mathcal{L}_{2}(H, K)$ and $\int_{0}^{T}\|\Phi(s)\|_{\mathcal{L}_{2}(H, K)}^{2} \mathrm{~d} s<\infty$ a.s. In this case $\int_{0}^{T} \Phi(s) \mathrm{d} W(s)$ is a well defined random variable with values in $K$ and

$$
\int_{0}^{T} \Phi(s) \mathrm{d} W(s)=\sum_{i=1}^{\infty} \int_{0}^{T} \Phi(s) e_{i} \mathrm{~d} \beta_{i}(s) .
$$

Moreover, if $\mathbb{E}\left(\int_{0}^{T}\|\Phi(s)\|_{\mathcal{L}_{2}(H, K)}^{2} \mathrm{~d} s\right)<\infty$, then

$$
\mathbb{E}\left(\int_{0}^{T} \Phi(s) \mathrm{d} W(s)\right)=0,
$$


and

$$
\mathbb{E}\left(\left\|\int_{0}^{T} \Phi(s) \mathrm{d} W(s)\right\|_{K}^{2}\right)=\mathbb{E}\left(\int_{0}^{T}\|\Phi(s)\|_{\mathcal{L}_{2}(H, K)}^{2} \mathrm{~d} s\right) .
$$

We will consider below expressions of the form $\int_{0}^{t} \psi(s) Q^{1 / 2} d W(s)$. These are then square integrable random variables in $K$ with zero average if

$$
\mathbb{E}\left(\int_{0}^{T}\left\|\psi(s) Q^{1 / 2}\right\|_{\mathcal{L}_{2}(H, K)}^{2} \mathrm{~d} s\right)=\mathbb{E} \int_{0}^{T} \operatorname{Tr}\left(\psi^{*}(s) Q \psi(s)\right) \mathrm{d} s<\infty .
$$

The solution of equation (2.4) can be written explicitly in terms of stochastic integrals. In order that these are well defined, we assume throughout this paper that there exist real numbers $\alpha>0$ and $\min (\alpha-1,0) \leq \beta \leq \alpha$ such that

$$
\sum_{n=1}^{\infty} \lambda_{n}^{-\alpha}=\left\|A^{-\alpha / 2}\right\|_{\mathcal{L}_{2}(H)}^{2}=\operatorname{Tr}\left(A^{-\alpha}\right)<\infty
$$

and

$$
A^{\beta} Q \in \mathcal{L}(H) .
$$

Condition (2.27) implies that $Q$ is a bounded operator from $H$ into $D\left(A^{\beta}\right)$. By interpolation, we deduce immediately that for any $\lambda \in[0,1], A^{\lambda \beta} Q^{\lambda} \in \mathcal{L}(H)$ and

$$
\left\|A^{\lambda \beta} Q^{\lambda}\right\|_{\mathcal{L}(H)} \leq\left\|A^{\beta} Q\right\|_{\mathcal{L}(H)}^{\lambda} .
$$

Example 2.3. If one considers the equations described in the introduction where $A$ is the Laplace operator with Dirichlet boundary conditions, it is well known that (2.26) holds for $\alpha>d / 2$.

We have the following result.

Proposition 2.4. Assume that (2.26), (2.27) hold and

$$
1-\alpha+\beta>0 \text {. }
$$

Then there exists a unique Gaussian stochastic process which is the weak solution (in the PDE sense) of (2.4) continuous in time with values in $L^{2}(\Omega, H)$. It is given by the formula which holds a.s. in $H$ :

$X_{t}=e^{-t A} x+\int_{0}^{t} e^{-(t-s) A} Q^{1 / 2} \mathrm{~d} W(s)=e^{-t A} x+\sum_{i=1}^{\infty}\left(\int_{0}^{t} e^{-(t-s) \lambda_{i}} \mathrm{~d} \beta_{i}(s)\right) Q^{1 / 2} e_{i}$.

Proof. By Theorem 5.4, p. 121 in [5], it is sufficient to see that the stochastic integral makes sense in $H$, i.e.

$$
\int_{0}^{t}\left\|e^{-(t-s) A} Q^{1 / 2}\right\|_{\mathcal{L}_{2}(H)}^{2} \mathrm{~d} s=\int_{0}^{t} \operatorname{Tr}\left(e^{-(t-s) A} Q e^{-(t-s) A}\right) \mathrm{d} s<\infty,
$$

for any $t \in[0, T]$. We use (2.9) to estimate the Hilbert-Schmidt norm:

$$
\begin{aligned}
\left\|e^{-(t-s) A} Q^{1 / 2}\right\|_{\mathcal{L}_{2}(H)} & \leq\left\|A^{\beta / 2} Q^{1 / 2}\right\|_{\mathcal{L}(H)}\left\|A^{-\alpha / 2}\right\|_{\mathcal{L}_{2}(H)}\left\|e^{-(t-s) A} A^{(-\beta+\alpha) / 2}\right\|_{\mathcal{L}(H)} \\
& \leq c(t-s)^{-1 / 2(-\beta+\alpha)}
\end{aligned}
$$

by (2.22), (2.26) and (2.28). The conclusion follows since $-\beta+\alpha<1$. 


\section{WEAK CONVERGENCE OF AN IMPLICIT SCHEME}

3.1. Setting of the problem and main result. In this section, we state the weak approximation result on the full discretization of (2.4).

We first describe the numerical scheme. Let $N \geq 1$ be an integer and $\left\{V_{h}\right\}_{h>0}$ the family of finite element spaces introduced in Section 2. Let $\Delta t=T / N$ and $t_{n}=n \Delta t, 0 \leq n \leq N$. For any $h>0$ and any integer $n \leq N$, we seek $X_{h}^{n}$, an approximation of $X_{t_{n}}$, such that for any $v_{h}$ in $V_{h}$ :

$$
\left(X_{h}^{n+1}-X_{h}^{n}, v_{h}\right)+\Delta t\left(A X_{h}^{n+\theta}, v_{h}\right)=\left(Q^{1 / 2} W\left(t_{n+1}\right)-Q^{1 / 2} W\left(t_{n}\right), v_{h}\right),
$$

with the initial condition

$$
\left(X_{h}^{0}, v_{h}\right)=\left(x, v_{h}\right), \quad \forall v_{h} \in V_{h},
$$

where

$$
X_{h}^{n+\theta}=\theta X_{h}^{n+1}+(1-\theta) X_{h}^{n}
$$

with

$$
1 / 2<\theta \leq 1
$$

Recall that the $\theta$-method defines a strongly stable scheme only when $\theta \in(1 / 2,1]$. For $\theta \leq 1 / 2$, a CFL condition is necessary.

Then (3.30)-3.31) can be rewritten as

$$
\begin{gathered}
X_{h}^{n+1}-X_{h}^{n}+\Delta t A_{h} X_{h}^{n+\theta}=\sqrt{\Delta t} P_{h} Q^{1 / 2} \chi^{n+1}, \\
X_{h}^{0}=P_{h} x
\end{gathered}
$$

where

$$
\chi^{n+1}=\frac{1}{\sqrt{\Delta t}}(W((n+1) \Delta t)-W(n \Delta t)),
$$

and where we recall that $P_{h}: H \rightarrow V_{h}$ is the $H$-orthogonal projector. Hence $\left\{\chi^{n}\right\}_{n \geq 0}$ is a sequence of independent and identically distributed Gaussian random variables. The main result of this paper is stated below.

Theorem 3.1. Let $\varphi \in C_{b}^{2}(H)$, i.e. a twice differentiable real-valued functional defined on $H$ whose first and second derivatives are bounded. Let $\alpha>0$ and $\beta \geq 0$ be such that (2.26), (2.27) and (2.29) hold. Let $T \geq 1$ and $\left\{X_{t}\right\}_{t \in[0, T]}$ be the $H$-valued stochastic process solution of (2.4) given by Proposition 2.4. For any $N \geq 1$, let $\left\{X_{h}^{n}\right\}_{0 \leq n \leq N}$ be the solution of the scheme (3.33)-(3.34). Then for any $\gamma<1-\alpha+\beta \leq 1$, there exists a constant $C=C(T, \varphi, \gamma,|x|)>0$ which does not depend on $h$ and $N$ such that the following inequality holds:

$$
\left|\mathbb{E} \varphi\left(X_{h}^{N}\right)-\mathbb{E} \varphi\left(X_{T}\right)\right| \leq C\left(h^{2 \gamma}+\Delta t^{\gamma}\right),
$$

where $\Delta t=T / N \leq 1$.

3.2. Proof of Theorem 3.1. The scheme (3.33)-(3.34) can be rewritten as

$$
X_{h}^{n}=S_{h, \Delta t}^{n} P_{h} x+\sqrt{\Delta t} \sum_{k=0}^{n-1} S_{h, \Delta t}^{n-k-1}\left(I+\theta \Delta t A_{h}\right)^{-1} P_{h} \chi^{k+1}, \quad 0 \leq n \leq N,
$$

where we have set for any $h>0$ and $N \geq 1$ :

$$
S_{h, \Delta t}=\left(I+\theta \Delta t A_{h}\right)^{-1}\left(I-(1-\theta) \Delta t A_{h}\right) .
$$

We split the proof into several steps. 
Step 1: We introduce discrete and semi-discrete auxiliary schemes which will be useful for the proof of Theorem 3.1.

First, for any $h>0$, let $\left\{X_{h}(t)\right\}_{t \in[0, T]}$ be the $V_{h}$-valued stochastic process solution of the following finite dimensional stochastic partial differential equation

$$
\mathrm{d} X_{h, t}+A_{h} X_{h, t} \mathrm{~d} t=P_{h} Q^{1 / 2} \mathrm{~d} W(t), \quad X_{h, 0}=P_{h} x .
$$

It is straightforward to see that $X_{h, t}$ can be written as

$$
X_{h, t}=S_{h}(t) P_{h} x+\int_{0}^{t} S_{h}(t-s) P_{h} Q^{1 / 2} \mathrm{~d} W(s) .
$$

The last stochastic integral is well defined since

$$
t \mapsto \operatorname{Tr}\left(\left(S_{h}(t) P_{h} Q^{1 / 2}\right)^{\star}\left(S_{h}(t) P_{h} Q^{1 / 2}\right)\right)
$$

is integrable on $[0, T]$.

We introduce also the following $V_{h}$-valued stochastic process

$$
Y_{h, t}=S_{h}(T-t) X_{h, t}, \quad t \in[0, T],
$$

which is the solution of the following drift-free finite dimensional stochastic differential equation

$$
\mathrm{d} Y_{h, t}=S_{h}(T-t) P_{h} Q^{1 / 2} \mathrm{~d} W(t), \quad Y_{h, 0}=S_{h}(T) P_{h} x .
$$

Its discrete counterpart is given by

$$
\begin{aligned}
Y_{h}^{n} & =S_{h, \Delta t}^{N-n} X_{h}^{n} \\
& =S_{h, \Delta t}^{N} P_{h} x+\sqrt{\Delta t} \sum_{k=0}^{n-1} S_{h, \Delta t}^{N-k-1}\left(I+\theta \Delta t A_{h}\right)^{-1} P_{h} Q^{1 / 2} \chi^{k+1},
\end{aligned}
$$

for $0 \leq n \leq N$.

Eventually, we consider a time continuous interpolation of $Y_{h}^{n}$ which is the $V_{h^{-}}$ valued $\left\{\mathcal{F}_{t}\right\}$-adapted stochastic process $\widetilde{Y}_{h, t}$ defined by

$$
\tilde{Y}_{h, t}=S_{h, \Delta t}^{N} P_{h} x+\int_{0}^{t} \sum_{n=0}^{N-1} S_{h, \Delta t}^{N-n-1}\left(I+\theta \Delta t A_{h}\right)^{-1} \mathbf{1}_{n}(s) P_{h} Q^{1 / 2} \mathrm{~d} W(s),
$$

where $\mathbf{1}_{n}$ denotes the function $\mathbf{1}_{\left[t_{n}, t_{n+1}\right)}$.

It is easy to see that for any $t \in[0, T]$ and $n$ such that $t \in\left[t_{n}, t_{n+1}\right)$, we have

$$
\widetilde{Y}_{h, t}=Y_{h}^{n}+S_{h, \Delta t}^{N-n-1}\left(I+\theta \Delta t A_{h}\right)^{-1} P_{h} Q^{1 / 2}\left(W(t)-W\left(t_{n}\right)\right) .
$$

Step 2: Splitting of the error.

Now let $\varphi \in C_{b}^{2}(H)$. The error $\mathbb{E} \varphi\left(X_{h}^{N}\right)-\mathbb{E} \varphi\left(X_{T}\right)$ can be split into two terms:

$$
\begin{aligned}
\mathbb{E} \varphi\left(X_{h}^{N}\right)-\mathbb{E} \varphi\left(X_{T}\right) & =\mathbb{E} \varphi\left(X_{h}^{N}\right)-\mathbb{E} \varphi\left(X_{h, T}\right)+\mathbb{E} \varphi\left(X_{h, T}\right)-\mathbb{E} \varphi\left(X_{T}\right) \\
& =A+B .
\end{aligned}
$$

The term $A$ contains the error due to the time discretization and will be estimated uniformly with respect to $h$. The term $B$ contains the spatial error.

Step 3: Estimate of the time discretization error. 
Let us now estimate the time error uniformly with respect to $h$. In order to do this, we consider the solution $v_{h}:[0, T] \times V_{h} \rightarrow \mathbb{R}$ of the following deterministic finite dimensional Cauchy problem:

$$
\left\{\begin{array}{l}
\frac{\partial v_{h}}{\partial t}(t, y)=\frac{1}{2} \operatorname{Tr}\left(\left(S_{h}(T-t) P_{h} Q^{1 / 2}\right)^{\star} D^{2} v_{h}(t, y)\left(S_{h}(T-t) P_{h} Q^{1 / 2}\right)\right) \\
v_{h}(0)=\varphi
\end{array}\right.
$$

We have the following classical representation of the solution of (3.42) at any time $t \in[0, T]$ and for any $y \in V_{h}$ (see for instance [6]):

$$
v_{h}(T-t, y)=\mathbb{E} \varphi\left(y+\int_{t}^{T} S_{h}(T-s) P_{h} Q^{1 / 2} \mathrm{~d} W(s)\right) .
$$

It follows easily that

$$
\left\|v_{h}(t)\right\|_{C_{b}^{2}(H)} \leq\|\varphi\|_{C_{b}^{2}(H)}, t \in[0, T] .
$$

Now, the estimate of the time error relies mainly on the comparison of Itô's formula applied successively to $t \mapsto v_{h}\left(T-t, Y_{h, t}\right)$ and $t \mapsto v_{h}\left(T-t, \widetilde{Y}_{h, t}\right)$. First, by construction, $t \mapsto v_{h}\left(T-t, Y_{h, t}\right)$ is a martingale. Indeed, Itô's formula gives

$$
\mathrm{d} v_{h}\left(T-t, Y_{h, t}\right)=\left(D v_{h}\left(T-t, Y_{h, t}\right), S_{h}(T-t) P_{h} Q^{1 / 2} \mathrm{~d} W(t)\right) .
$$

Therefore,

$v_{h}\left(T-t, Y_{h, t}\right)=v_{h}\left(T, S_{h}(T) P_{h} x\right)+\int_{0}^{t}\left(D v_{h}\left(T-s, Y_{h, s}\right), S_{h}(T-s) P_{h} Q^{1 / 2} \mathrm{~d} W(s)\right)$.

Taking $t=T$ and the expectation implies since $x$ is deterministic

$$
\mathbb{E} \varphi\left(X_{h, T}\right)=v_{h}\left(T, S_{h}(T) P_{h} x\right) .
$$

On the contrary, $t \mapsto v_{h}\left(T-t, \widetilde{Y}_{h, t}\right)$ is not a martingale. Nevertheless, applying Itô's formula gives, thanks to (3.40),

$$
\begin{aligned}
& \mathbb{E} v_{h}\left(0, \widetilde{Y}_{h, T}\right)=\mathbb{E} v_{h}\left(T, \widetilde{Y}_{h, 0}\right)-\mathbb{E} \int_{0}^{T} \frac{\partial v_{h}}{\partial t}\left(T-t, \widetilde{Y}_{h, t}\right) \mathrm{d} t \\
& +\frac{1}{2} \mathbb{E} \int_{0}^{T} \sum_{n=0}^{N-1} \operatorname{Tr}\left[\left(S_{h, \Delta t}^{N-n-1} T_{h, \Delta t} P_{h} Q^{1 / 2}\right)^{\star} D^{2} v_{h}\left(S_{h, \Delta t}^{N-n-1} T_{h, \Delta t} P_{h} Q^{1 / 2}\right)\right] \mathbf{1}_{n}(t) \mathrm{d} t,
\end{aligned}
$$

where here and in equations (3.46), (3.47) below, $D^{2} v_{h}$ is evaluated at $\left(T-t, \widetilde{Y}_{h, t}\right)$. Also we have set

$$
T_{h, \Delta t}=\left(I+\theta \Delta t A_{h}\right)^{-1} .
$$

Thanks to (3.42) we get

$$
\begin{aligned}
\mathbb{E} \varphi\left(X_{h}^{N}\right) & =v_{h}\left(T, S_{h, \Delta t}^{N} P_{h} x\right) \\
(3.46) & +\frac{1}{2} \mathbb{E} \int_{0}^{T} \sum_{n=0}^{N-1} \operatorname{Tr}\left[\left(S_{h, \Delta t}^{N-n-1} T_{h, \Delta t} P_{h} Q^{1 / 2}\right)^{\star} D^{2} v_{h}\left(S_{h, \Delta t}^{N-n-1} T_{h, \Delta t} P_{h} Q^{1 / 2}\right)\right. \\
& \left.-\left(S_{h}(T-t) P_{h} Q^{1 / 2}\right)^{\star} D^{2} v_{h}\left(S_{h}(T-t) P_{h} Q^{1 / 2}\right)\right] \mathbf{1}_{n}(t) \mathrm{d} t .
\end{aligned}
$$


At last, the comparison between (3.45) and (3.46) leads to the following decomposition of the time error $A$,

$$
\begin{aligned}
\mathbb{E} \varphi\left(X_{h}^{N}\right) & -\mathbb{E} \varphi\left(X_{h, T}\right)=v_{h}\left(T, S_{h, \Delta t}^{N} P_{h} x\right)-v_{h}\left(T, S_{h}(T) P_{h} x\right) \\
(3.47) & +\frac{1}{2} \mathbb{E} \int_{0}^{T} \sum_{n=0}^{N-1} \operatorname{Tr}\left[\left(S_{h, \Delta t}^{N-n-1} T_{h, \Delta t} P_{h} Q^{1 / 2}\right)^{\star} D^{2} v_{h}\left(S_{h, \Delta t}^{N-n-1} T_{h, \Delta t} P_{h} Q^{1 / 2}\right)\right. \\
& \left.-\left(S_{h}(T-t) P_{h} Q^{1 / 2}\right)^{\star} D^{2} v_{h}\left(S_{h}(T-t) P_{h} Q^{1 / 2}\right)\right] \mathbf{1}_{n}(t) \mathrm{d} t, \\
& =I+I I .
\end{aligned}
$$

The term $I$ is the pure deterministic part of the time error. Thanks to the representation (3.43), we have

$$
I \leq\|\varphi\|_{\mathcal{C}_{b}^{1}(H)}\left\|S_{h}(T) P_{h}-S_{h, \Delta t}^{N} P_{h}\right\|_{\mathcal{L}(H)}|x| .
$$

Thanks to (3.32) it is possible to bound $I$ uniformly with respect to $h$. More precisely, we have (e.g. see Theorem 1.1, p. 921 in [23])

$$
\begin{aligned}
\left\|\left(S_{h}(N \Delta t)-S_{h, \Delta t}^{N}\right) P_{h}\right\|_{\mathcal{L}(H)} & =\sup _{i \geq 1}\left|e^{-N \lambda_{i, h} \Delta t}-F^{N}\left(\lambda_{i, h} \Delta t\right)\right| \\
& \leq \sup _{z \geq 0}\left|e^{-N z}-F^{N}(z)\right| \\
& \leq \frac{C}{N}=C \frac{\Delta t}{T} .
\end{aligned}
$$

We have used the following notation:

$$
F(z)=\frac{1-(1-\theta) z}{1+\theta z}, z>0 .
$$

We obtain

$$
I \leq C\|\varphi\|_{\mathcal{C}_{b}^{1}(H)} \frac{\Delta t}{T}|x| .
$$

Let us now see how to estimate the term $I I$. First, using the symmetry of $D^{2} v_{h}$, we rewrite the trace term as

$$
\begin{aligned}
& \operatorname{Tr}\left(\left(S_{h, \Delta t}^{N-n-1} T_{h, \Delta t} P_{h} Q^{1 / 2}-S_{h}(T-t) P_{h} Q^{1 / 2}\right)^{\star}\right. \\
& \left.\quad D^{2} v_{h}\left(S_{h, \Delta t}^{N-n-1} T_{h, \Delta t} P_{h} Q^{1 / 2}-S_{h}(T-t) P_{h} Q^{1 / 2}\right)\right) \\
& +2 \operatorname{Tr}\left(\left(S_{h}(T-t) P_{h} Q^{1 / 2}\right)^{\star} D^{2} v_{h}\left(S_{h, \Delta t}^{N-n-1} T_{h, \Delta t} P_{h} Q^{1 / 2}-S_{h}(T-t) P_{h} Q^{1 / 2}\right)\right) \\
& =a_{n}(t)+b_{n}(t),
\end{aligned}
$$

for $t \in\left[t_{n}, t_{n+1}\right)$.

Now let $\alpha>0$ and $\beta \geq 0$ such that (2.26) and (2.27) hold with $0<1-\alpha+\beta \leq 1$. Let $\gamma>0$ and $\gamma_{1}>0$ such that $0<\gamma<\gamma_{1}<1-\alpha+\beta \leq 1$. 
We first estimate the term $a_{n}(t)$. We use (2.6), (2.7), (2.8), (3.44) and (2.9) to obtain for $t \in\left[t_{n}, t_{n+1}\right)$

$$
\begin{aligned}
& a_{n}(t) \leq\left\|v_{h}(T-t)\right\|_{C_{b}^{2}(H)} \\
& \times \operatorname{Tr}\left(\left(S_{h, \Delta t}^{N-n-1} T_{h, \Delta t} P_{h} Q^{1 / 2}-S_{h}(T-t) P_{h} Q^{1 / 2}\right)^{\star}\right. \\
& \left.\quad\left(S_{h, \Delta t}^{N-n-1} T_{h, \Delta t} P_{h} Q^{1 / 2}-S_{h}(T-t) P_{h} Q^{1 / 2}\right)\right) \\
& \leq\|\varphi\|_{C_{b}^{2}(H)}\left\|\left(S_{h, \Delta t}^{N-n-1} T_{h, \Delta t}-S_{h}(T-t)\right) P_{h} Q^{1 / 2}\right\|_{\mathcal{L}_{2}(H)}^{2} \\
& \leq\|\varphi\|_{C_{b}^{2}(H)}\left\|\left(S_{h, \Delta t}^{N-n-1} T_{h, \Delta t}-S_{h}(T-t)\right) A_{h}^{\left(1-\gamma_{1}\right) / 2} P_{h}\right\|_{\mathcal{L}_{(H)}}^{2} \\
& \left\|A_{h}^{\left(\gamma_{1}-1\right) / 2} P_{h} Q^{1 / 2}\right\|_{\mathcal{L}_{2}(H)}^{2} .
\end{aligned}
$$

Let us set for $t \in\left[t_{n}, t_{n+1}\right)$

$$
\begin{aligned}
M_{n}(t) & =\left\|\left(S_{h, \Delta t}^{N-n-1} T_{h, \Delta t}-S_{h}(T-t)\right) A_{h}^{\left(1-\gamma_{1}\right) / 2} P_{h}\right\|_{\mathcal{L}(H)} \\
& =\sup _{1 \leq i \leq I(h)}\left|\frac{F^{N-n-1}\left(\lambda_{i, h} \Delta t\right)}{1+\theta \lambda_{i, h} \Delta t}-e^{-\lambda_{i, h}(T-t)}\right| \lambda_{i, h}^{\left(1-\gamma_{1}\right) / 2} .
\end{aligned}
$$

Using similar techniques as for the proof of the strong order (see e.g. 28]), we have the following bound, for $n<N-1$,

$$
M_{n}(t) \leq C \Delta t^{\gamma / 2}((N-n-1) \Delta t)^{-\left(1-\gamma_{1}+\gamma\right) / 2}, \text { for } t \in\left[t_{n}, t_{n+1}\right),
$$

where here and below $C$ denotes a constant which depends only on $\gamma_{1}, \gamma,\|\varphi\|_{C_{b}^{2}(H)}$, $\left\|A^{\beta} Q\right\|_{\mathcal{L}(H)}$ and $\operatorname{Tr}\left(A^{-\alpha}\right)$. In particular, these constants do not depend on $h$ or $\Delta t$. The proof is postponed to the appendix at the end of this article.

We then estimate the last factor in (3.50). Using (2.9), we get

$$
\left\|A_{h}^{\left(\gamma_{1}-1\right) / 2} P_{h} Q^{1 / 2}\right\|_{\mathcal{L}_{2}(H)} \leq\left\|A_{h}^{\left(\gamma_{1}-1\right) / 2} P_{h} A^{-\beta / 2}\right\|_{\mathcal{L}_{2}(H)}\left\|A^{\beta / 2} Q^{1 / 2}\right\|_{\mathcal{L}(H)} .
$$

Using (2.13) and (2.17), we have

$$
\begin{aligned}
\left\|A_{h}^{\left(\gamma_{1}-1\right) / 2} P_{h} A^{-\beta / 2}\right\|_{\mathcal{L}_{2}(H)}^{2} & =\sum_{i \in \mathbb{N}}\left|A_{h}^{\left(\gamma_{1}-1\right) / 2} P_{h} A^{-\beta / 2} e_{i}\right|^{2} \\
& \leq \sum_{i \in \mathbb{N}}\left|A_{h}^{-1 / 2} P_{h} A^{-\beta / 2} e_{i}\right|^{2\left(1-\gamma_{1}\right)}\left|P_{h} A^{-\beta / 2} e_{i}\right|^{2 \gamma_{1}} \\
& \leq \sum_{i \in \mathbb{N}}\left|A^{-1 / 2-\beta / 2} e_{i}\right|^{2\left(1-\gamma_{1}\right)}\left|A^{-\beta / 2} e_{i}\right|^{2 \gamma_{1}} \\
& =\sum_{i \in \mathbb{N}} \lambda_{i}^{-\left(1-\gamma_{1}+\beta\right)} .
\end{aligned}
$$

We deduce from $1-\gamma_{1}+\beta>\alpha$ and (2.28) that

$$
\left\|A_{h}^{\left(\gamma_{1}-1\right) / 2} P_{h} A^{-\beta / 2}\right\|_{\mathcal{L}_{2}(H)}^{2} \leq \lambda_{1}^{1-\gamma_{1}+\beta-\alpha}\left\|A^{\beta} Q\right\|_{\mathcal{L}(H)}^{1 / 2} \operatorname{Tr}\left(A^{-\alpha}\right) .
$$

Plugging this and (3.52) into (3.50) yields for $n<N-1, t \in\left[t_{n}, t_{n+1}\right)$ :

$$
a_{n}(t) \leq C \Delta t^{\gamma}((N-n-1) \Delta t)^{-\left(1-\gamma_{1}+\gamma\right)} .
$$

For $n=N-1$, for $t \in\left[t_{N-1}, T\right]$, we derive similarly,

$$
\begin{aligned}
a_{N-1}(t) & \leq\|\varphi\|_{C_{b}^{2}(H)}\left\|\left(T_{h, \Delta t}-S_{h}(T-t)\right) A_{h}^{\left(1-\gamma_{1}\right) / 2}\right\|_{\mathcal{L}(H)}^{2}\left\|A_{h}^{\left(1-\gamma_{1}\right) / 2} P_{h} Q^{1 / 2}\right\|_{\mathcal{L}_{2}(H)}^{2} \\
& \leq C\left(\left\|T_{h, \Delta t} A_{h}^{\left(1-\gamma_{1}\right) / 2}\right\|_{\mathcal{L}(H)}^{2}+\left\|S_{h}(T-t) A_{h}^{\left(1-\gamma_{1}\right) / 2}\right\|_{\mathcal{L}(H)}^{2}\right) \\
& \leq C\left(\Delta t^{\gamma_{1}-1}+(T-t)^{\gamma_{1}-1}\right) \\
& \leq C(T-t)^{\gamma_{1}-1} .
\end{aligned}
$$


Concerning $b_{n}$, we write for $t \in\left[t_{n}, t_{n+1}\right)$,

$$
\begin{aligned}
& b_{n}(t)=2 \operatorname{Tr}\left(\left(S_{h}(T-t) A_{h}^{\left(1-\gamma_{1}\right) / 2} A_{h}^{\left(\gamma_{1}-1\right) / 2} P_{h} Q^{1 / 2}\right)^{\star} D^{2} v_{h}\right. \\
& \left.\quad\left(S_{h, \Delta t}^{N-n-1} T_{h, \Delta t}-S_{h}(T-t)\right) A_{h}^{\left(1-\gamma_{1}\right) / 2} A_{h}^{\left(\gamma_{1}-1\right) / 2} P_{h} Q^{1 / 2}\right) \\
& \leq\|\varphi\|_{C_{b}^{2}(H)}\left\|S_{h}(T-t) A_{h}^{\left(1-\gamma_{1}\right) / 2}\right\|_{\mathcal{L}(H)}\left\|\left(S_{h, \Delta t}^{N-n-1} T_{h, \Delta t}-S_{h}(T-t)\right) A_{h}^{\left(1-\gamma_{1}\right) / 2}\right\|_{\mathcal{L}(H)} \\
& \quad \times\left\|A_{h}^{\left(\gamma_{1}-1\right) / 2} P_{h} Q^{1 / 2}\right\|_{\mathcal{L}_{2}(H)}^{2} .
\end{aligned}
$$

Using a similar argument as above, we prove

$$
\left\|S_{h}(T-t) A_{h}^{\left(1-\gamma_{1}\right) / 2}\right\|_{\mathcal{L}(H)} \leq C(T-t)^{\left(\gamma_{1}-1\right) / 2}
$$

and, for $n<N-1, t \in\left[t_{n}, t_{n+1}\right)$,

$\left\|\left(S_{h, \Delta t}^{N-n-1} T_{h, \Delta t}-S_{h}(T-t)\right) A_{h}^{\left(1-\gamma_{1}\right) / 2}\right\|_{\mathcal{L}(H)} \leq C \Delta t^{\gamma}((N-n-1) \Delta t)^{-\left(\left(1-\gamma_{1}\right) / 2+\gamma\right)}$

so that for $n<N-1, t \in\left[t_{n}, t_{n+1}\right)$ :

$$
b_{n}(t) \leq C \Delta t^{\gamma}((N-n-1) \Delta t)^{-\left(1-\gamma_{1}+\gamma\right)} .
$$

For $n=N-1, t \in\left[t_{N-1}, T\right]$, we have

$$
\begin{aligned}
b_{N-1}(t) & \leq\|\varphi\|_{C_{b}^{2}(H)}\left\|S_{h}(T-t) A_{h}^{\left(1-\gamma_{1}\right) / 2}\right\|_{\mathcal{L}\left(V_{h}\right)} \\
& \times\left\|\left(T_{h, \Delta t}-S_{h}(T-t)\right) A_{h}^{\left(1-\gamma_{1}\right) / 2} P_{h}\right\|_{\mathcal{L}\left(V_{h}\right)}\left\|A_{h}^{\left(1-\gamma_{1}\right) / 2} P_{h} Q^{1 / 2}\right\|_{\mathcal{L}_{2}\left(H, V_{h}\right)}^{2} \\
& \leq C(T-t)^{\gamma_{1}-1} .
\end{aligned}
$$

We are now ready to bound $I I$ in (3.47). Indeed, (3.53), (3.54), (3.55), (3.56) imply

$$
\begin{aligned}
I I \leq & C \int_{0}^{T-\Delta t} \sum_{n=0}^{N-2} \Delta t^{\gamma}((N-n-1) \Delta t)^{-\left(1-\gamma_{1}+\gamma\right)} \mathbf{1}_{n}(t) \mathrm{d} t \\
& +C \int_{T-\Delta t}^{T}(T-t)^{\gamma_{1}-1} \mathrm{~d} t \\
\leq & C \Delta t^{\gamma} .
\end{aligned}
$$

Then, plugging (3.49) and (3.57) into (3.47) we obtain

$$
\begin{aligned}
|A| & \leq C\|\varphi\|_{\mathcal{C}_{b}^{1}(H)} \frac{\Delta t}{T}|x|+C \Delta t^{\gamma} \\
& \leq C \Delta t^{\gamma},
\end{aligned}
$$

for $T \geq 1, \Delta t \leq 1$.

Step 4: Estimate of the space discretization error.

Let us now estimate the spatial error $B$. The method is essentially the same as above: we use the Kolmogorov equation associated to the transformed process $Y_{t}$.

We consider the following linear parabolic equation on $H$ : (3.59)

$$
\frac{\partial v}{\partial t}(t, x)=\frac{1}{2} \operatorname{Tr}\left(\left(S(T-t) Q^{1 / 2}\right)^{\star} D^{2} v(t, x)\left(S(T-t) Q^{1 / 2}\right)\right), \quad t>0, \quad x \in H,
$$

together with the initial condition

$$
v(0, x)=\varphi(x), \quad x \in H
$$


where $v$ is a real-valued function of $t$ and $x \in H$. We have the following representation of $v$ (see e.g. [6], Chapter 3) at time $t \in[0, T]$ and any $y \in H$ :

$$
v(T-t, y)=\mathbb{E} \varphi\left(y+\int_{t}^{T} S(T-s) Q^{1 / 2} \mathrm{~d} W(s)\right) .
$$

We apply the Itô formula to $t \mapsto v\left(T-t, Y_{t}\right)$ and $t \mapsto v\left(T-t, Y_{h, t}\right)$. We subtract the resulting equations and obtain

$$
\begin{aligned}
& \mathbb{E} \varphi\left(X_{T}\right)-\mathbb{E} \varphi\left(X_{h, T}\right)=v(T, S(T) x)-v\left(T, S_{h}(T) P_{h} x\right) \\
& +\frac{1}{2} \mathbb{E} \int_{0}^{T} \operatorname{Tr}\left[\left(S_{h}(T-t) P_{h} Q^{1 / 2}\right)^{\star} D^{2} v\left(T-t, Y_{h, t}\right)\left(S_{h}(T-t) P_{h} Q^{1 / 2}\right)\right] \mathrm{d} t \\
& -\frac{1}{2} \mathbb{E} \int_{0}^{T} \operatorname{Tr}\left[\left(S(T-t) Q^{1 / 2}\right)^{\star} D^{2} v\left(T-t, Y_{h, t}\right)\left(S(T-t) Q^{1 / 2}\right)\right] \mathrm{d} t .
\end{aligned}
$$

The first term on the right hand side of (3.61) is the deterministic spatial error which can be bounded thanks to (2.24) (with $s=0$ and $q=2 \gamma<2$ ) and (3.60). We obtain:

$$
\left|v(T, S(T) x)-v\left(T, S_{h}(T) P_{h} x\right)\right| \leq \kappa_{1}\|\varphi\|_{C_{b}^{1}(H)} h^{2 \gamma} T^{-\gamma}|x| .
$$

For the second term, we use the symmetry of $D^{2} v$ and write

$$
\begin{aligned}
& \operatorname{Tr}\left[\left(S_{h}(T-t) P_{h} Q^{1 / 2}\right)^{\star} D^{2} v\left(S_{h}(T-t) P_{h} Q^{1 / 2}\right)\right. \\
& \left.-\left(S(T-t) Q^{1 / 2}\right)^{\star} D^{2} v\left(S(T-t) Q^{1 / 2}\right)\right] \\
& =\operatorname{Tr}\left[\left(S_{h}(T-t) P_{h} Q^{1 / 2}-S(T-t) Q^{1 / 2}\right)^{\star} D^{2} v\left(S_{h}(T-t) P_{h} Q^{1 / 2}-S(T-t) Q^{1 / 2}\right)\right] \\
& +2 \operatorname{Tr}\left[\left(S(T-t) Q^{1 / 2}\right)^{\star} D^{2} v\left(S_{h}(T-t) P_{h} Q^{1 / 2}-S(T-t) Q^{1 / 2}\right)\right] \\
& =a+b,
\end{aligned}
$$

where here and below $D^{2} v$ is evaluated at $\left(T-t, Y_{h, t}\right)$.

Let $\gamma>0$ and $\gamma_{1}>0$ such that $0<\gamma<\gamma_{1}<1-\alpha+\beta \leq 1$. Thanks to (2.6), (2.7) and (3.60), we get the following bounds:

$$
\begin{aligned}
b= & 2 \operatorname{Tr}\left(S(T-t) D^{2} v\left(S_{h}(T-t) P_{h}-S(T-t)\right) Q\right) \\
= & 2 \operatorname{Tr}\left(A^{\gamma_{1}-1-\beta} A^{1-\gamma_{1}} S(T-t) D^{2} v\left(S_{h}(T-t) P_{h}-S(T-t)\right) Q A^{\beta}\right) \\
\leq & 2\left\|\left(S_{h}(T-t) P_{h}-S(T-t)\right)\right\|_{\mathcal{L}(H)}\|\varphi\|_{\mathcal{C}_{b}^{2}(H)}\left\|A^{1-\gamma_{1}} S(T-t)\right\|_{\mathcal{L}(H)} \\
& \times\left\|Q A^{\beta}\right\|_{\mathcal{L}(H)} \operatorname{Tr}\left(A^{1-\gamma_{1}-\beta}\right) .
\end{aligned}
$$

Then, owing to (2.22), (2.24) (with $s=0$ and $q=2 \gamma<2$ ), we obtain

$$
b \leq C h^{2 \gamma}(T-t)^{-\left(1+\gamma-\gamma_{1}\right)}
$$

where again $C$ denotes a constant which depends only on $\gamma_{1}, \gamma,\|\varphi\|_{C_{b}^{2}(H)}$, $\left\|A^{\beta} Q\right\|_{\mathcal{L}(H)}$ and $\operatorname{Tr}\left(A^{-\alpha}\right)$, but not on $h$ or $\Delta t$. 
In regard to $a$, we get first, thanks to (2.7), (2.28) and (3.60),

$$
\begin{aligned}
a \leq & \|\varphi\|_{C_{b}^{2}(H)} \operatorname{Tr}\left(Q\left(S_{h}(T-t) P_{h}-S(T-t)\right)^{\star}\left(S_{h}(T-t) P_{h}-S(T-t)\right)\right) \\
= & \|\varphi\|_{C_{b}^{2}(H)}\left\|Q^{1 / 2}\left(S_{h}(T-t) P_{h}-S(T-t)\right)\right\|_{\mathcal{L}_{2}(H)}^{2} \\
\leq & \|\varphi\|_{C_{b}^{2}(H)}\left\|Q^{1 / 2} A^{\beta / 2}\right\|_{\mathcal{L}(H)}^{2}\left\|A^{\left(1-\gamma_{1}\right) / 2}\left(S_{h}(T-t) P_{h}-S(T-t)\right)\right\|_{\mathcal{L}(H)}^{2} \\
& \times\left\|A^{-\left(1-\gamma_{1}+\beta\right) / 2}\right\|_{\mathcal{L}_{2}(H)}^{2} \\
\leq & C\left\|A^{\left(1-\gamma_{1}\right) / 2}\left(S_{h}(T-t) P_{h}-S(T-t)\right)\right\|_{\mathcal{L}(H)}^{2},
\end{aligned}
$$

where we have used (2.28). If $\gamma_{1}+\gamma \geq 1$, we interpolate (2.24) with $q=\left(\gamma+\gamma_{1}-\right.$ $1) / \gamma, s=0$ and (2.25) with $s^{\prime}=0, q^{\prime}=1$ and get

$$
\left\|A^{\left(1-\gamma_{1}\right) / 2}\left(S_{h}(T-t) P_{h}-S(T-t)\right)\right\|_{\mathcal{L}(H)} \leq C h^{\gamma}(T-t)^{-\left(1-\gamma_{1}+\gamma\right) / 2} .
$$

If $\gamma_{1}+\gamma<1$, we interpolate (2.24) with $q=0, s=0$ and (2.25) with $s^{\prime}=0, q^{\prime}=1$ and get

$$
\left\|A^{\left(1-\gamma_{1}\right) / 2}\left(S_{h}(T-t) P_{h}-S(T-t)\right)\right\|_{\mathcal{L}(H)} \leq C h^{1-\gamma_{1}}(T-t)^{-\left(1-\gamma_{1}\right)} .
$$

We use again an interpolation argument to get (3.66)

$$
\begin{aligned}
& \left\|A^{\left(1-\gamma_{1}\right) / 2}\left(S_{h}(T-t) P_{h}-S(T-t)\right)\right\|_{\mathcal{L}(H)} \\
& \leq C\left\|\left(S_{h}(T-t) P_{h}-S(T-t)\right)\right\|_{\mathcal{L}(H)}^{\gamma_{1}}\left\|A^{1 / 2}\left(S_{h}(T-t) P_{h}-S(T-t)\right)\right\|_{\mathcal{L}(H)}^{1-\gamma_{1}} \\
& \leq C\left(\left\|A^{1 / 2} S_{h}(T-t) P_{h}\right\|_{\mathcal{L}(H)}+\left\|A^{1 / 2} S(T-t)\right\|_{\mathcal{L}(H)}\right)^{1-\gamma_{1}} \\
& \leq C(T-t)^{\left(1-\gamma_{1}\right) / 2}
\end{aligned}
$$

thanks to (2.22) for $A$ and $A_{h}$ and (2.15). A further interpolation between (3.65) and (3.66) gives

$$
\begin{gathered}
\left\|A^{\left(1-\gamma_{1}\right) / 2}\left(S_{h}(T-t) P_{h}-S(T-t)\right)\right\|_{\mathcal{L}(H)} \\
\leq C h^{\lambda\left(1-\gamma_{1}\right)}(T-t)^{-\left(1-\gamma_{1}\right)(\lambda+(1-\lambda) / 2)} .
\end{gathered}
$$

Taking $\lambda=\gamma /\left(1-\gamma_{1}\right)$ shows that (3.64) again holds for $\gamma_{1}+\gamma \leq 1$.

We deduce

$$
a \leq C h^{2 \gamma}(T-t)^{-1+\gamma_{1}-\gamma} .
$$

Plugging (3.62), (3.63) and (3.68) into (3.61) leads to, after time integration which is relevant since $1-\gamma_{1}+\gamma<1$,

$$
|B| \leq C h^{2 \gamma}
$$

for $T \geq 1$.

Conclusion. Gathering (3.58) and (3.69) in (3.41) ends the proof of Theorem 3.1 


\section{APPENDIX}

We now prove the estimate (3.52) on $M_{n}, n<N-1$, defined in (3.51).

Below, $t \in\left[t_{n}, t_{n+1}\right)$. We proceed as follows:

$$
\begin{aligned}
M_{n}(t) & \leq \sup _{1 \leq i \leq I(h)}\left|\frac{F^{N-n-1}\left(\lambda_{i, h} \Delta t\right)-e^{-\lambda_{i, h}\left(T-t_{n+1}\right)}}{1+\theta \lambda_{i, h} \Delta t}\right| \lambda_{i, h}^{\left(1-\gamma_{1}\right) / 2} \\
& +\sup _{1 \leq i \leq I(h)}\left|\frac{e^{-\lambda_{i, h}\left(T-t_{n+1}\right)}-e^{-\lambda_{i, h}(T-t)}}{1+\theta \lambda_{i, h} \Delta t}\right| \lambda_{i, h}^{\left(1-\gamma_{1}\right) / 2} \\
& +\sup _{1 \leq i \leq I(h)} e^{-\lambda_{i, h}(T-t)}\left(1-\frac{1}{1+\theta \Delta t \lambda_{i, h}}\right) \lambda_{i, h}^{\left(1-\gamma_{1}\right) / 2} \\
& =a_{1}+a_{2}+a_{3} .
\end{aligned}
$$

Thanks to (3.48) with $N$ replaced by $N-n-1$ we get

$$
\begin{aligned}
a_{1} & \leq \frac{\kappa_{4}}{N-n-1} \sup _{i \geq 1}\left(\frac{\lambda_{i, h}^{\left(1-\gamma_{1}\right) / 2}}{1+\theta \lambda_{i, h} \Delta t}\right) \\
& \leq \frac{\kappa_{4} \Delta t^{\left(\gamma_{1}-1\right) / 2}}{(N-n-1)^{\left(1-\gamma_{1}+\gamma\right) / 2}} \sup _{i \geq 1}\left(\frac{\left(\lambda_{i, h} \Delta t\right)^{\left(1-\gamma_{1}\right) / 2}}{1+\theta \lambda_{i, h} \Delta t}\right) \\
& \leq \frac{\kappa_{4} \Delta t^{\gamma / 2}}{((N-n-1) \Delta t)^{\left(1-\gamma_{1}+\gamma\right) / 2}} .
\end{aligned}
$$

Indeed, since $\left(1-\gamma_{1}+\gamma\right) / 2<1,(N-n-1) \geq(N-n-1)^{\left(1-\gamma_{1}+\gamma\right) / 2}$. In the same way, we have

$$
\begin{aligned}
a_{2} & \leq \sup _{i \geq 1}\left(\frac{1-e^{-\left(t_{n+1}-t\right) \lambda_{i, h}}}{\left(1+\theta \Delta t \lambda_{i, h}\right)} \lambda_{i, h}^{\left(1-\gamma_{1}\right) / 2} e^{-(N-n-1) \Delta t \lambda_{i, h}}\right) \\
& \leq C(\gamma) \Delta t^{\gamma / 2} \sup _{i \geq 1}\left(\lambda_{i, h}^{\left(1-\gamma_{1}+\gamma\right) / 2} e^{-(N-n-1) \Delta t \lambda_{i, h}}\right) \\
& \leq \frac{C\left(\gamma, \gamma_{1}\right) \Delta t^{\gamma / 2}}{((N-n-1) \Delta t)^{\left(1-\gamma_{1}+\gamma\right) / 2}},
\end{aligned}
$$

where we have used that $\left|t-t_{n+1}\right| \leq \Delta t$ and the inequality $\left|e^{-x}-e^{-y}\right| \leq C_{\gamma}|x-y|^{\gamma / 2}$ and (2.23). Eventually, similar computations lead to

$$
\begin{aligned}
a_{3} & \leq \Delta t^{\gamma / 2} \sup _{i \geq 1}\left(\frac{\theta\left(\Delta t \lambda_{i, h}\right)^{1-\gamma / 2}}{1+\theta \Delta t \lambda_{i, h}} \lambda_{i, h}^{\left(1-\gamma_{1}+\gamma\right) / 2} e^{-(T-t) \lambda_{i, h}}\right) \\
& \leq C\left(\gamma, \gamma_{1}\right) \Delta t^{\gamma / 2}(T-t)^{-\left(1-\gamma_{1}+\gamma\right) / 2} \\
& \leq C\left(\gamma, \gamma_{1}\right) \Delta t^{\gamma}((N-n-1) \Delta t)^{-\left(1-\gamma_{1}+\gamma\right) / 2},
\end{aligned}
$$

where we have used again the inequality (2.23).

Gathering these three estimates in (3.70) yields (3.52), for $n<N-1$.

\section{REFERENCES}

[1] E.J. ALLEN, S.J. NOVOSEL, Z. ZHANG, Finite element and difference approximation of some linear stochastic partial differential equations, Stochastics Stochastics Rep., 64 (1998), 117-142. MR1637047 (99d:60067)

[2] J. H. BRAMBLE, A. H. SCHATZ, V. THOMÉE, L. B. WAHLBIN, Some convergence estimates for semidiscrete Galerkin type approximations for parabolic equations, SIAM J. Numer. Anal. 14 (1977), 218-241. MR0448926 (56:7231) 
[3] E. BUCKWAR, T. SHARDLOW, Weak approximation of stochastic differential delay equations, IMA J. Numer. Anal. 25 (2005), 57-86. MR2110235 (2006a:65012)

[4] P. G. CIARLET, The Finite Element Method for Elliptic Problems, North-Holland, Amsterdam-London-New-York, 1978. MR0520174 (58:25001)

[5] G. DA PRATO and J. ZABCZYK, Stochastic Equations in Infinite Dimensions, Encyclopedia of mathematics and its applications 44, Cambridge University Press, 1992. MR:1207136 (95g:60073)

[6] G. DA PRATO and J. ZABCZYK, Second Order Partial Differential Equations in Hilbert Spaces, London Mathematical Society, Lecture Note Series 293, Cambridge University Press, 2002. MR 1985790(2004e:47058)

[7] A.M. DAVIE, J.G. GAINES, Convergence of numerical schemes for the solution of parabolic stochastic partial differential equations, Math. Comp. 70 (2001), 121-134 MR1803132 (2001h:65012)

[8] A. DE BOUARD, A. DEBUSSCHE, Weak and strong order of convergence of a semi discrete scheme for the stochastic Nonlinear Schrodinger equation, Appl. Math. and Optim., $\mathbf{5 4}$ (2006), 369-399. MR.2268663 (2008g:60208)

[9] M. GEISSERT, M. KOVACS, S. LARSSON, Rate of weak convergence of the finite element method for the stochastic heat equation with additive noise, Preprint.

[10] I. C. GOKHBERG, M. G. KREĬN, Introduction to the theory of linear nonselfadjoint operators in Hilbert space, Amer. Math. Soc., Providence, RI, 1970.

[11] W. GRECKSCH, P.E. KLOEDEN, Time-discretised Galerkin approximations of parabolic stochastic PDEs, Bull. Austral. Math. Soc. 54 (1996), 79-85. MR1402994 (97g:60080)

[12] I. GYÖNGY, Lattice approximations for stochastic quasi-linear parabolic partial differential equations driven by space-time white noise. I, Potential Anal. 9 (1998), 1-25. MR1644183 (99j:60091)

[13] I. GYONGY, Lattice approximations for stochastic quasi-linear parabolic partial differential equations driven by space-time white noise. II, Potential Anal. 11 (1999), 1-37 MR1699161 (2000g:60106)

[14] I. GYONGY, A. MILLET, On discretization schemes for stochastic evolution equations, Potential Analysis 23 (2005), 99-134. MR2139212 (2006a:60115)

[15] I. GYÖNGY, A. MILLET, Rate of Convergence of Implicit Approximations for stochastic evolution equations, Stochastic Differential Equations: Theory and Applications. A volume in Honor of Professor Boris L. Rosovskii, Interdisciplinary Mathematical Sciences, Vol. 2, World Scientific (2007), 281-310.

[16] I. GYÖNGY, A. MILLET, Rate of convergence of space time approximations for stochastic evolution equations, Preprint (2007).

[17] I. GYÖNGY, D. NUALART, Implicit scheme for stochastic parabolic partial differential equations driven by space-time white noise, Potential Anal. 7 (1997), 725-757. MR:1480861 (98m:60097)

[18] E. HAUSENBLAS, Approximation for semilinear stochastic evolution equations in Banach spaces, Journal in Comp. and Appl. Math., 147 (2002), 485-516. MR1933610 (2003j:35338)

[19] E. HAUSENBLAS, Approximation for semilinear stochastic evolution equations, Potential Analysis, 18 (2003), 141-186. MR:1953619 (2003m:60167)

[20] E. HAUSENBLAS, Weak approximation of stochastic partial differential equations. in Stochastic analysis and related topics VIII. Silivri workshop, Progress in Probability. U. Capar and A. Üstünel editors. Basel: Birkhäuser, 2003. MR2189620 (2006k:60114)

[21] C. JOHNSON, S. LARSSON, V. THOMÉE, L. B. WALHBIN, Error estimates for spatially discrete approximations of semilinear parabolic equations with non smooth initial data, Math. Comput., 49, (1987), 331-357. MR906175 (88k:65100)

[22] P.E. KLOEDEN, E. PLATEN, Numerical solution of stochastic differential equations, Applications of Mathematics, 23, Springer-Verlag, New York, 1992. MR1214374 (94b:60069)

[23] M.-N. LE ROUX, Semidiscretization in Time for Parabolic Problems, Math. Comput., 33 (1979), 919-931. MR528047 (80f:65101)

[24] G. LORD, J. ROUGEMONT, A Numerical Scheme for stochastic PDEs with Gevrey Regularity, IMA J. Num. Anal., 24 (2004), 587-604. MR2094572 (2005d:60102)

[25] A. MILLET, P.L. MORIEN, On implicit and explicit discretization schemes for parabolic SPDEs in any dimension, Stoch. Proc. and Appl. 115 (2005), no 7, 1073-1106. MR 2147242 (2006b:60141) 
[26] G. N. MILSTEIN, Numerical integration of stochastic differential equations, Mathematics and its Applications, 313, Kluwer Academic Publishers, Dordrrecht, 1995. MR.1335454 (96e:65003)

[27] G. N. MILSTEIN, M. V. TRETYAKOV, Stochastic numerics for mathematical physics, Scientific Computation series, Springer-Verlag, 2004. MR2069903 (2005f:60004)

[28] J. PRINTEMS, On the discretization in time of parabolic stochastic partial differential equations, Math. Model. and Numer. Anal., 35 (2001), 1055-1078. MR.1873517(2002j:60116)

[29] T. SHARDLOW, Numerical methods for stochastic parabolic PDEs, Numer. Funct. Anal. Optim., 20 (1999), 121-145. MR.1683281 (2000g:65004)

[30] G. STRANG, G.J. FIX, An Analysis of the Finite Element Method, Prentice-Hall, Englewood Cliffs, 1973. MR0443377 (56:1747)

[31] D. TALAY, Probabilistic numerical methods for partial differential equations: elements of analysis, Probabilistic models for nonlinear partial differential equations (Montecatini Terme, 1995), 148-196, Lecture Notes in Math., 1627, Springer, Berlin, 1996. MR1431302 (98j:60092)

[32] V. THOMÉE, Galerkin Finite Element Methods for Parabolic Problems, Springer-Verlag, 1997. MR1479170 (98m:65007)

[33] Y. YAN, Galerkin finite element methods for stochastic parabolic partial differential equations, SIAM J. Numer. Anal., 43 (2005), 1363-1384. MR2182132 (2007a:65013)

[34] Y. YAN, Semidiscrete Galerkin approximation for a linear stochastic parabolic partial differential equation driven by an additive noise, BIT 44 (2004), 829-847. MR2211047 (2007c:60065)

[35] J.B. WALSH Finite element methods for parabolic stochastic PDE's, Potential Anal. 23 (2005), 1-43. MR2136207 (2006b:60155)

irmaR et EnS de Cachan, antenne de Bretagne, Campus de Ker Lann, avenue Robert Schumann, 35170 Bruz, France

E-mail address: arnaud.debussche@bretagne.ens-cachan.fr

Laboratoire d'Analyse et de Mathématiques Appliquées, CNRS UMR 8050, Université de Paris XiI, 61, avenue du Général de Gaulle, 94010 Créteil, France

E-mail address: printems@univ-paris12.fr 